\title{
Corrientes del pensamiento arquitectónico para el siglo XXI
}

\section{Currents of Architectural Thought for the $21^{\text {st }}$ century}

\author{
Prof. Juan R. Castillo Molina, Dr. Eng. \\ Coordinador de Innovación y Arquitectura \\ Universidad Nacional Pedro Henríquez Ureña (UNPHU). \\ Santo Domingo, República Dominicana \\ jcamolina@yahoo.com
}

Fecha de recepción: 16 de septiembre de 2019

Fecha de aceptación: 15 de octubre de 2019

Fecha de publicación: 1 de enero de 2020

\section{RESUMEN}

En tiempos de virtualidad desenfrenada que amenazan con debilitar la realidad táctil de la Arquitectura es importante establecer lo que es unívoco en ella: su capacidad de definir lugares usando elementos tangibles. Arquitectura es un hecho objetual, y aunque responde a un proceso intelectual, el producto es un objeto modelado o construido que históricamente ha tenido la capacidad de sobrepasar teorías establecidas. La profesión necesita continuar aumentando el valor de la obra exponencialmente debido a su potencial tangible. El objetivo del presente artículo es establecer lo unívoco en Arquitectura como visión significativa para su conceptualización y práctica. La metodología de análisis está basada en cómo los nuevos conceptos ideospacio, objetospacio, dataspacio y luminospacio convergen en el campo de lo tangible. Como resultado, se encontró lo unívoco como la línea fundamental para fomentar corrientes de pensamiento arquitectónico para el siglo XXI.

Palabras claves: arquitectura, objetual, proceso, tangible, unívoco.

\begin{abstract}
In times when virtual applications threat to weak the tactile reality of Architecture, the univocal fact of Architecture must be established based on its capacity to define tangible places. Although the process of Architecture is intellectual, it remains objectual, for the results are modeled or built with the historical ability to overcome established theories. The objective of this article is to establish the univocal fact of Architecture as a significant vision to conceptualize and practice. The analysis method is based on how new concepts as ideospacio, objetospacio, dataspacio and luminospacio converge in the tangible field. As a result, it is found that the
\end{abstract}


univocal fact of architecture is the baseline to strengthen new lines of architectural thought for the XXI century.

Keywords: architecture, objectual, process, space, tangible, univocal.

\section{Introducción}

El presente artículo se inspira en el llamado de reiniciar la profesión cuidándose de «no tratar de institucionalizar rebeldías vanguardistas, sino de revisar lo inoperante, transformar lo que obstruye el progreso de la humanidad e inaugurar una era que supere el concepto mismo de civilización» (Castillo Molina, 2019, p.55). Lo unívoco en Arquitectura se ejerce a través de la relación entre idea y objeto, la cual es automática y evita que cualquier filosofía, teoría o proceso efímero distraiga el potencial tectónico de la Arquitectura, revelando la evidencia tangible como algo irrefutable.

El objetivo de este artículo es establecer lo unívoco en Arquitectura como visión significativa para la conceptualización, práctica y nuevas maneras de pensamiento de la misma, y esto se abordará con una metodología de análisis basado en nuevos conceptos propositivos que surgen y convergen en el campo de lo tangible, denotando que la profesión va más allá de la inmediatez de las Tecnologías de Información y Comunicación (TIC), asunto pertinente al tema central de la presente publicación.

El presente artículo no está en contra de las teorías y filosofías que han tratado de definir la profesión históricamente, sino que está enfocado en cómo el potencial tangible de la Arquitectura garantiza un verdadero valor cultural. El siglo pasado fue un tiempo de contradicciones y rebeliones (las vanguardias), pero hoy hay una vasta cantidad de cultura e información para manejar esos vestigios turbulentos y movernos hacia la optimización del conocimiento evitando el ardid del conflicto, y aumentando la capacidad de imaginación y lógica reflejada en el contexto tangible circundante. No hay necesidad de enfrentamientos sino de potenciar y buscarle sentido a la sobreabundancia de conocimiento.
Y si el pasado milenio fue un tiempo de luchas intelectuales fértiles, ahora es el momento de desarrollar los inventos derivados de esa época, $\mathrm{y}$ en cuyo proceso hay que seguir edificando las ciudades. A pesar de que los medios de exploración virtuales están modificando la percepción del medioambiente construido, es una era donde la presencia del objeto, como hecho físico, juega un rol significativo en el uso cuidadoso de los recursos limitados que ofrece el planeta.

Cuando este artículo habla sobre Arquitectura se refiere a todo diseño o construcción con presencia tangible, y no a la Filosofía, Historia o Teoría de la Arquitectura. Igualmente, se está consciente de que la profesión tiene la capacidad de estar basada en poesía, pero ese campo requiere una especial atención más allá del espacio destinado a este ensayo. Es necesario aclarar que el concepto de Arquitectura presentado aquí es un marco de referencia para entender el concepto de lo unívoco y no condena la diversidad, ya que se introduce en una época cuando cada arquitecto tiene la libertad de elaborar su propia definición de la profesión.

Los siguientes capítulos están titulados usando vocablos híbridos inventados especialmente para este artículo (ideospacio, objetospacio, dataspacio y luminospacio), que consisten en la unión de dos palabras donde ninguna de los dos tiene prioridad, sino que forman una unidad conceptual que combina lo mejor de ambos significados - convergencia de ideas. Todos esos nuevos conceptos tienen como denominador común la palabra «espacio», por ser la expresión universal de la Arquitectura.

\section{Ideospacio}

El ideospacio es el espacio imaginado en arquitectura. El vocablo está escrito combinando el término ideo (derivado de idea) y espacio. 
Es cierto que todo espacio arquitectónico viene con una idea, aunque en el caso de la arquitectura vernácula la repetición prima sobre el ingenio, pero en el ideospacio la idea está intencionalmente expuesta. La idea es la construcción mental del intelecto, que por su carácter inmaterial, puede exponer toda la obra o una parte solamente, revelando su belleza en cada momento. La idea es infinita, y puede ser discutida sin importar el tiempo arquitectónico. Es también recurrente o puede repetirse sin perder su potencia debido a su naturaleza abstracta; es fuente pura de inspiración y es un medio genuino de formalizaciones intelectuales (Asimov, 1959).

El ideospacio trae consigo una visión que transforma la idea en artefacto. La invención de la rueda fue revolucionaria no por la rueda en sí, sino probablemente por la idea de desplazar cosas más rápido y con menor fricción que los antiguos rodillos, por ejemplo. Obviamente, el invento en sí es importante pero es la idea la que lo hace crucial. Igualmente, una idea singular tiene la capacidad de ramificarse en múltiples formas y provocar una cadena de inventos. De la proyección bélica de alcanzar un objetivo a grandes distancias surge la invención del cohete, y consecuentemente emergió la conquista del universo, abriendo paso a la invención de los satélites, robots exploradores, hábitats en órbita, naves estelares, estaciones espaciales y demás productos de la carrera espacial, demostrando que la idea legitima el descubrimiento. Más allá de ser un logro, es un recurso y un detonante, se necesita estructurarlas, ponerlas a prueba y generar más. Así como el conocimiento es usado para crear las ideas, que crecen a medida que evolucionan las civilizaciones.

El ideospacio aumenta su potencial en el mundo de las vanguardias. La importancia de las estéticas vanguardistas radica en las imágenes que las forman, aquellas que maduran y finalmente detonan nuevas ideas de cambio. Las vanguardias, como campos experimentales y fértiles donde todo es posible, son el ecosistema del ideospacio. El mundo de la construcción tiene otra forma de producir ideas inesperadas a través de los imprevistos y accidentes, pues «analizando la negatividad del accidente de un objeto se puede desarrollar este mismo objeto para perfeccionarlo, para humanizarlo y civilizarlo [...].» (Rial Ungaro, 2003, p.86).

El ideospacio puede ser aplicable y aplicado. El primero (aplicable) es aquel aún en incubación que espera el momento y los recursos óptimos para convertirse en un hecho. El segundo (aplicado) existe, está presente y listo para ser reutilizado dentro del mismo campo que la originó o fuera del mismo. Ambos pueden impactar al observador a través de lo tangible como medio idóneo para establecer el ideospacio y examinarlo eventualmente en la Filosofía. Por ejemplo, la Historia o Teoría de la Arquitectura lo necesitan para validar datos y reconstruir pensamientos que acompañen la evolución de la profesión.

Diferentes medios sirven de canal de comunicación del ideospacio, y de acuerdo al medio escogido se puede básicamente entender en dos formatos: abstractos y concretos. En el primero se desmaterializa, reside en la mente de quien las crea, y necesita la imaginación para darle forma a toda la visión. En el segundo se transmite por medios específicos como películas, pinturas, objetos y música, y en este set se muestra estructurado y listo para ser valorado; el mundo visual (la imagen) es un campo mudo que completa la dinámica entre lo objetual y lo pensado -zona fértil de las ideas.

En Arquitectura, el ideospacio es concretizado en proyectos (edificios, paisajes artificiales o ciudades completas) los cuales usan otros medios paralelos para diseminarlo como revistas, el cine o la literatura, teniendo todos en común que la imaginación es la energía principal de la invención. Esto demuestra que si el ideospacio está bien pensado puede pasar de un formato a otro sin perder su relevancia, lo cual es una de las razones para que acompañe el proceso de pensamiento arquitectónico, donde se puede navegar entre ciencia y arte sin perder la potencia original. 
El ideospacio inspira tecnologías. Su descubrimiento genera qué tipo de herramientas pueden materializarlo. También ocurre lo inverso, pues se espera que el surgimiento de nuevas tecnologías genere un nuevo ideospacio, estableciendo qué tipo de ideas se producen con cuál tipo de herramientas. Hay casos donde ambos procesos se convierten en uno, por ejemplo, la inmediatez de la fabricación digital en Arquitectura aumenta la velocidad de configuración del ideospacio, y su materialización es casi instantánea; el tiempo de producción se reduce debido al cálculo de prueba y error automatizado por los programas de simulación digital. Esta experiencia lleva la imaginación del arquitecto a una zona creativa ilimitada aumentando al mismo tiempo la precisión tangible de sus propuestas.

\section{Objetospacio}

El objetospacio es la forma geométrica física del espacio arquitectónico que funciona a la manera de un ideograma japonés donde signo y significado son inseparables. Es aquello que garantiza que lo tangible tenga presencia unívoca en el proceso de pensamiento arquitectónico. El vocablo está escrito combinando los términos objeto y espacio. El objetospacio evidencia cómo el ideospacio puede ser objetivo y útil para implementarse en proyectos reales. Su dimensión es tangible y llega a su zenit en la obra construida, donde el observador arma fácilmente la idea total de conjunto, guiándose de cualidades táctiles que aumentan el nivel de realidad a través de textura y luz.

En el proceso de diseño existen la realidad virtual y las maquetas como herramientas para explorar el objetospacio. Recientemente, la realidad virtual se ha convertido en una forma de aprehensión casi real de la Arquitectura, pero el observador necesita un equipo adicional (casco y guantes) y restringir su radio de acción a una porción espacial pequeña $(3.00 \times 3.00$ metros cuadrados aproximadamente) ya que todavía los movimientos son principalmente simulados en el cerebro humano (Kurzweil, 2005).
En el proceso de diseño, los modelos (maquetas físicas) son utilizados como «herramientas para ver» (Lambert, 2001, p.215); los arquitectos pueden fácilmente visualizar una imagen concreta mentalmente y la completan en el modelo, el cual le ayuda a confirmar o cuestionar su visión aún a través de un modelo conceptual. Aunque las maquetas consumen tiempo y no son recomendadas para diseñar todo hasta el último detalle, son necesarias para probar las cualidades formales de la arquitectura desde un ángulo palpable. Es cierto que en el modelo el observador no llega a la inmersión total como lo haría en VR (virtual reality $=$ realidad virtual), pero en ambos casos la imaginación entra en juego y se convierte en el recurso intelectual por excelencia del arquitecto como pensador.

El objetospacio evidencia que la Arquitectura no es una idea, sino un producto socioeconómico de las civilizaciones. Aún cuando esté en forma dibujada o modelada sigue estando presente, listo para que las escuelas de arquitectura lo utilicen para mejorar la manera en la que se piensa el medioambiente construido, y para abrir conversaciones fructíferas entre academia y práctica -lo físico es el medio de convergencia entre la planificación y ejecución del espacio. Lo que está construido fuera del aula no es el producto del arquitecto sino de las complejidades reales de la profesión. La mente del arquitecto es completamente diferente, pues está llena de infinitas soluciones que hacen posible hasta la más arriesgada idea. La potencia de pensamiento se reduce cuando pasa al ejercicio; si se trabaja solo con lo empírico, la conceptualización esencial se pierde en el proceso. Para eliminar la desconexión entre los dos campos (teoría y práctica) no puede hacerse con las posiciones que se tienen hoy (academia muy teórica vs. práctica muy empírica); por un lado se debe escribir y debatir sobre la importancia de lo tangible en las escuelas (objetospacio), y por otro se debe culturizar sobre la relevancia de la idea arquitectónica en la práctica (ideospacio) para avanzar la profesión hacia una unificación intelectual y técnica. 
El objetospacio pone a prueba el proceso de pensamiento y experimentación de la Arquitectura. Aún en las representaciones fotográficas (medio bi-dimensional), el objetospacio se siente físico y tectónico, y por ser un medio relativamente estático (al menos que se recorra y deforme a alta velocidad) deja lugar para las sorpresas que la imaginación trae consigo, dando tiempo para la reflexión indispensable que toda arquitectura exige.

\section{Dataspacio}

El dataspacio explica cómo la Arquitectura incorpora los dos estados de información y materia simultáneamente. El vocablo está escrito combinando el término data (derivado de la Informática, datos) y espacio. La Arquitectura tiene una relación fluida con las Tecnologías de Información y Comunicación (TIC), pues su cualidad tangible, aquello que la convierte en unívoca, necesita las TIC para llegar a lugares inesperados. Para lograrlo, debe convertirse en datos binarios (dematerializarse), para luego ser recuperada en sistemas de navegación virtuales; es decir, Arquitectura, un medioambiente construido; necesita ser transformada en información para poder difundirse a través de aplicaciones, aparatos y visores que permitan un acercamiento real a estructuras que por alguna razón serían inaccesibles. Este proceso de dematerialización y recuperación es tradicional en Arquitectura, pues su base conceptual (la idea) necesita convertirse primero en pensamiento (datos intangibles) y luego recuperarse en forma física a través de dibujos, modelos o edificios, demostrando que el paso hacia el uso de las TIC ha sido natural. Pero ese proceso viene con la advertencia de que mientras las aplicaciones se vuelven obsoletas la Arquitectura debe continuar siendo un repositorio de cultura universal (aún en momentos sin electricidad), y es lo tangible lo que le asegura esto.

Las TIC permiten manipular los datos de la humanidad, y Arquitectura, como contenedor cultural, atesora vasta información sobre las civilizaciones (Cole, 2006). En los edificios de la antigüedad convergen el conocimiento empírico y la experimentación debido a la búsqueda de soluciones ambiciosas en términos espaciales que expresen el Zeitgeist -el espíritu del tiempo- (Neumeyer,1991), compitiendo con las de tiempos anteriores, aumentando la escala de las construcciones y multiplicando los procedimientos para llevarlas a cabo. Las opciones tectónicas del siglo XXI se han sumado a esos fenómenos históricos exigiendo la necesidad de crear bases de datos que ayuden a los historiadores y teóricos a manejar la casi inconmensurable fuente de conocimiento de la profesión. Las TIC son cruciales para manipular todos esos documentos, y sobre todo, para hacerlos accesibles al público interesado, fortaleciendo la red multidisciplinaria entre ejercicio y academia.

Una parte importante de las TIC son los programas de modelado y renderizado arquitectónicos (representación tridimensional con texturas casi realistas), los cuales están evolucionando hacia una imagen gemela de la realidad misma. Hoy día, los nuevos programas promocionan sus productos tomando frases prestadas de la Física que utilizan como «espectralmente correcto» o «profundidad de campo» para garantizar el acercamiento a lo táctil del espacio real («About Octane Render», 2019). Ese fenómeno confirma que el dataspacio es un aspecto importante para las TIC, pues lo táctil sigue siendo el vínculo inequívoco entre las abstracciones del autor y el sentido común del público general.

La Arquitectura, como invención humana, es una corriente de pensamiento en sí misma, y hoy día su novedad viene integrando las herramientas digitales de última generación dentro de sus procesos de planificación y materialización. Esos medios ofrecen un campo perfecto donde la estética (lo subjetivo) y el cálculo (la precisión) pueden coexistir, de forma que las propuestas no son exclusivamente imaginadas, sino programadas y materializadas debido al proceso computacional facilitado hoy día por las TIC y 
ejecutado a través del dataspacio, compactando lo multidisciplinario de la profesión en unidades que ocupan un espacio casi imperceptible (Reas, \& McWilliams, 2010).

\section{Luminospacio}

El luminospacio es un concepto híbrido que explica cómo el ambiente de un lugar depende de la calidad física del mismo a través de la luz. El vocablo está escrito combinando el término inventado lumino (derivado de lo lumínico y lo óptico) y espacio. Por estar conectado a lo tangible en Arquitectura, crea consciencia de los limitados que son los recursos que utiliza la profesión, e inspira la producción eficiente de diseños de alta calidad con el uso mínimo de materiales. Debido al desmedido uso de los recursos naturales, es necesario el empleo mínimo de materia prima para formar elementos arquitectónicos. La idea de reducir la cantidad de los recursos aprovechando las ventajas de las herramientas digitales de próxima generación puede proveer una Arquitectura que responda a los retos medioambientales que el presente siglo plantea; se deben reducir los gastos y la cantidad del material a utilizar, pero elevando la calidad arquitectónica de la propuesta (Rams, 2014). Hoy, la extravagancia arquitectónica domina la profesión exigiendo el empleo desmesurado de materiales, pero esa situación debe cambiar hacia una solución inteligente que sea alcanzada a través de la optimización. Finalmente, la obra se destacará por el diseño de alta calidad y no por el dramatismo estético que varios estilos contemporáneos insisten en establecer (Schumacher, 2008). Trabajar con ese tipo de eficiencia (uso mínimo del material) produce que el luminospacio se destaque más allá de la sobreabundancia tangible, y finalmente expanda la atmósfera que emana de la Arquitectura.

El luminospacio promulga una consciencia medioambiental. Al no tener necesidad de ornamentación abundante como ocurre tradicionalmente en la Arquitectura, elimina el exceso de objetos para enfocarse en la sensación espacial, logrando que el detalle se funda en la atmósfera del espacio arquitectónico dando paso a la pura aprehensión arquitectónica. Ludwig Hilberseimer, junto a otros exponentes de la Nueva Objetividad, apoyó las bases de una estética escueta a principios del siglo XX, influenciado por la crítica de Alfréd Kemény sobre la obra del escultor László Péri, que reflejaba «la economía de las formas mínimas; la tensión espacial producida por la extrema oposición de formas mínimas; potencia masiva; determinación objetiva afinada desasociadas de cualquier relación con la naturaleza» (Anderson, 2013, p.53). Lo que se conoce como Minimalismo también ha madurado esas ideas a través de la apariencia del no-ornamento en el diseño. El híbrido luminospacio evita hacer eco de esas posiciones, sino que las aprovecha para promocionar una consciencia medioambiental tomando lo reductivo como tema significante. El deterioro ambiental del mundo, y posiblemente de la órbita terrestre baja en un futuro cercano, es un asunto socio-económico complejo que va más allá de la Arquitectura, y debe analizarse en otra oportunidad para evitar exceder el espacio de la presente publicación.

El luminospacio acerca la Arquitectura y el cine, ambos medios culturales universales y representantes de las revoluciones estéticas de la humanidad. En las películas prevalece la potencia de la imagen y la cinematografía como recursos para transmitir la atmósfera del tema (Truffaut, 1974), algo que se compara con el impacto de la plástica y la cuarta dimensión en Arquitectura (el recorrido). Los filmes son una fuente inspiradora para los arquitectos, debido a que los mismos revelan otra vida de los espacios, es decir, cada escena ocurre en un lugar que es usado y habitado artísticamente, más allá de lo que las filosofías o teorías pudieran plantear, algo que revela cómo la acción ocurre dentro de lo tangible, estableciendo este último como lo unívoco en Arquitectura.

La Arquitectura es un campo de acción donde suceden cosas no exclusivamente para 
contemplarse, pues son precisamente esos límites físicos reales (tangibles) los que provocan que esas acciones sucedan; y es esa relación entre acción-objeto que detona ideas que resultan finalmente en otra nueva obra arquitectónica. Toda esa corporeidad genera atmósferas definidas por la forma geométrica, materiales luz y escala (Tedeschi, 1969), convirtiendo el luminospacio en la expresión poética palpable de la Arquitectura.

\section{Lo unívoco}

Lo unívoco en Arquitectura es su evidencia tangible, aquella que garantiza el valor de la obra a través de las cualidades físicas de la misma. Si está bien hecha no expirará, y disminuirá la necesidad de levantar nuevas edificaciones innecesarias, reduciendo la extracción de materia prima, y fomentando la utilización óptima de los limitados recursos del planeta.

Algunos arquitectos dan charlas sobre ideas y conceptos solamente, olvidando profundizar en los asuntos que hacen de la Arquitectura un hecho tangible, y omitiendo el valor objetual de la Arquitectura; ellos se enfocan en temas de críticas, teorías, economía y política, pero omiten la calidad tectónica que eventualmente afecta el medioambiente físico circundante.

Lo unívoco en Arquitectura equivale a lo palpable, ya sea construido, dibujado o modelado; aquel elemento cuyas propiedades se mantienen como la característica constante y universal de la profesión a través del tiempo. Aún en el Suprematismo (la supremacía de lo intangible) Kazimir Malevich hace uso de lo tangible para aclarar sus conceptos del no-objeto (Railing, 1990), e Ivan Leonidov continuó esas teorías para proyectar su Arquitectura experimental sin perder el propósito objetual de la obra (Magomedov, 2011).

Lo tangible como equivalente a lo unívoco en Arquitectura es el valor central de esta profesión. Abordar principalmente el objeto no se refiere a que el proceso de pensamiento se descuide, pues este último es el responsable del resultado palpable final. Aparte de referirse a lo externo y lo que provoca sensibilidad, lo tangible es importante para entender que la Arquitectura es un artículo físico que se experimenta visualmente en la mayoría de los casos. Hay exploraciones artísticas que usan la tecnología cibernética para producir una atmósfera cromática en personas con discapacidad visual, permitiendo que puedan explorar un espacio artificialmente, pero esos intentos aún están en incubación, dejando que las propiedades visuales todavía dominen la experiencia (Bryant, 2013). También, la granulometría, como visualización morfológica casi molecular en el proceso de diseño, descompone el objeto en componentes granulares, tratando de inaugurar una nueva estética donde lo unívoco (tangible) se perciba de acuerdo al orden y tamaño del grano (Carpo, 2017).

La Arquitectura no es autónoma del todo, pero si está bien diseñada pertenece a un no-tiempo y de alguna manera adquiere cierta autonomía que hace que valga la pena construirla hoy y usarse perfectamente en el mañana -así adquiere su valor. Por ejemplo, los planes de negocio, teorías y filosofías destacan el valor objetual de la Arquitectura solo durante el proceso, pues sus productos (enfoques) son otros: el plan negocio se enfoca en el éxito financiero, y las teorías y filosofías se enfocan en conceptos; es decir, las condiciones del presente son solo las bases de nuevos tipos de Arquitectura y no garantizan la vigencia de la obra en el futuro, por eso el objeto debe tener la capacidad de devenirse autónomo.

Se insiste en equivaler lo tangible como lo unívoco en Arquitectura para concientizar a los arquitectos (estudiantes y profesionales) de la realidad tectónica de la profesión, y de que estar conscientes del potencial tangible es estar conscientes de cuan finitas son las materias primas para construir. Aunque la Arquitectura no está hecha de materia solamente, sino de ideas y conceptos también, lo unívoco (lo tangible) posee la capacidad de resumir una compleja idea en medios finitos y desvelarla a través de una visión tectónica.

Pensar de manera objetual inspira a pensar en diseño, y éste último exige a cambio que la 
Arquitectura como evidencia de habitabilidad siga cuatro hechos que declaran lo tangible como unívoco en ella:

1. la Arquitectura es proyectada. La capacidad del arquitecto de imaginar el proyecto es insuficiente si no puede transformar esa visión en algo concreto y proyectarlo a una audiencia plural. En ese caso, la arquitectura deviene como una necesidad de ser producida para otros, generando representaciones gráficas y físicas que transforman el diseño en documentos recurrentes durante la etapa conceptual y promoción del producto final.

2. la Arquitectura es construida. Proyectarla solamente es insuficiente si se necesita evolucionarla. Aunque desde el pasado milenio la arquitectura proyectada ha tomado importancia en el discurso de la Historia y Teoría de la Arquitectura, tradicionalmente la posición de estas dos ramas están basadas en obras construidas como evidencia de las limitaciones y riesgos que todo hecho tangible plantea. Además, la necesidad de salir de las cavernas provocó la creación de ambientes controlados, y finalmente llegar a algo que fuera más allá de un refugio y se convirtiera en ejemplo físico de la idiosincrasia de su habitante.

3. la Arquitectura es ocupada. Construirla es irrelevante si carece de propósito, que en rasgos generales se reduce al acto de «habitar». Los niveles de habitabilidad de la Arquitectura aumentan al ritmo que incrementa la sofisticación de los estilos de vida, pero la cualidad de ser habitada es constante a través de todas las tipologías que paulatinamente surgen y cambian de acuerdo a la transformación de la vida humana.

4. la Arquitectura es reusada. El potencial de sobrevivir tiempos más allá de su planificación garantiza el potencial de ser reutilizada, en este caso, lo tangible le concede longevidad al hecho arquitectónico.

Si se está consciente de lo tangible como lo unívoco en Arquitectura, es menester cambiar la manera en que la profesión es investigada académicamente, y evolucionar hacia un proceso que disminuya textos pero que aumente la cantidad de gráficos, dibujos, modelos, fotografías $\mathrm{u}$ otra forma de representación que unifiquen pensamiento y diseño. Es necesario que las investigaciones y publicaciones sean hechas casi exclusivamente de manera gráfica, inaugurando un nuevo rigor académico equivalente al que tiene una publicación científica tradicional. Igualmente, el formato de redacción debe ser uno acorde con la manera no lineal en la que se formula el conocimiento arquitectónico. Se debe hacer un formato abierto que se estructure de acuerdo a los objetivos de la investigación específica, en vez de ajustar los resultados a un estándar que podría disminuir la potencia del artículo. El método científico aplica parcialmente a una profesión compleja y realmente multidisciplinaria como la Arquitectura. Hay que aprovechar la cualidad tangible de la profesión para lograr un acercamiento más profundo entre academia y práctica, pues los productos arquitectónicos significantes son hechos físicos y exigen que se tenga consciencia de ello en todo momento de la investigación. Eventualmente, los resultados deben estar enfocados hacia proyectos que puedan ser debatidos y comprobables bajo la realidad física de los contextos donde se proyecten los hallazgos de las investigaciones.

Pensar en lo unívoco sugiere que la Arquitectura posee una sola forma de entenderse, un solo significado, y aunque está claro que el estudio del significado pertenece a la Semiótica en Arquitectura, ese no es el sentido que se expone en el presente artículo. Si lo tangible, como evidencia del hecho construido y proyectado es universal, en ese sentido se descubre algo unívoco, algo que es determinante e inequívoco a través de las culturas y el tiempo.

Se pasó del ideospacio (formación de lo tangible) al objetospacio (tangible) y luego al luminospacio (atmósfera tangible) pasando por el dataspacio (documentación y transformación de lo tangible) como territorios intelectuales de lo unívoco. Se tiene en cuenta que cada uno de esos conceptos no suceden en el orden expuesto (no ocurren linealmente), pero se recomienda tomarlos en cuenta al momento de formular corrientes de pensamiento avanzadas para el presente milenio. 
Lo tangible, aunque esté compuesto de elementos que corresponden a una época específica, carece de la variable tiempo cuando se entiende a través de lo unívoco. Incorporarlo y estar consciente de ese concepto durante el proceso de investigación, diseño y práctica de la Arquitectura ayuda a eliminar los momentos de indecisión que la ciencia no puede confirmar en situaciones cuando la máquina falla, y le devolverá al arquitecto la confianza en la intuición (convergencia de experiencia y racionalismo) como recurso infalible que ha acompañado y seguirá acompañando la profesión a través de toda la historia.

\section{Referencias}

«About Octane Render». (2019). Recuperado en (12 septiembre 2019) en URL: https://home.otoy.com/render/ octane-render/

Anderson, R. (2013). Ludwig Hilberseimer: Metrópolis Architecture. Nueva York: GSAPP BOOKS.

Asimov, I. (1959). How Do People Get New Ideas. Recuperado el (12 septiembre 2019) en URL: https://www. technologyreview.com/s/531911/isaac-asimov-askshow-do-people-get-new-ideas/

Bryant, R. (2013). People Will Start Becoming Technology. Recuperado el (14 de septiembre 2019) en URL: https://www.dezeen.com/2013/11/20/interview-with-human-cyborg-neil-harbisson/

Carpo, M. (2017). The Second Digital Turn: Design Beyond Intelligence. Boston: MIT Press.

Castillo Molina, JR. (2019). Innovación y transferencia: la implementación del hallazgo. AULA Revista de Humanidades y Ciencias Sociales. Vol. 64. Número 4. Páginas 4656. enero-junio 2019. Santo Domingo: Amigo del Hogar.

Cole, E. (2006). La gramática de la Arquitectura. Madrid: Lisma Ediciones, S.L.

Kurzweil, R. (2005). The Singularity is Near: When Humans Trascend Biology. Nueva York: Penguin Books.
Lambert, P.(2001). Mies in America. Montréal: Canadian Centre for Architecture.

Magomedov, S. (2011). Heroes of the Avant-Garde. Ivan Leonidov. Moscow: M., Sergey Gordeev.

Railing, P.(1990). Suprematism: 34 drawings. London: Artists Bookworks.

Neumeyer, F. (1991). The Artless word: Mies van der Rohe on the Building Art. Cambridge: The MIT Press.

Rams, D. (2014). Less But Better. Hamburg: Jo Klatt Desig$\mathrm{n}+$ Design Verlag.

Reas, C. \& McWilliams, C. (2010). Form and Code in Design, Art, and Architecture. New York: Princeton Architectural Press.

Rial Ungaro, S. (2003). Paul Virilio y los límites de la velocidad. Madrid: Campo de Ideas SL.

Schumacher, P.(2008). Parametricism as Style- Parametricist Manifesto. Recuperado el (21 de agosto 2019) en URL: https://www.patrikschumacher.com/Texts/Parametricism\%20as\%20Style.htm

Tedeschi, E. (1969). Teoría de la Arquitectura. Buenos Aires: Ediciones Nueva Visión.

Truffaut, F. (1974). El cine según Hitchcok. Madrid: Alianza Editorial.

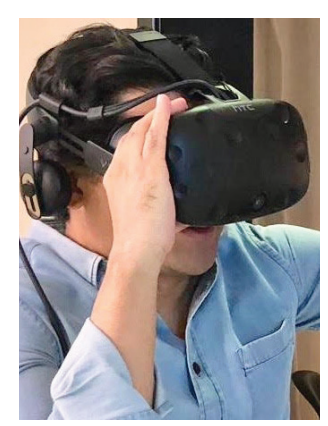

\section{Juan R. Castillo}

Arquitecto y docente interesado en procesos avanzados arquitectónicos. Egresado de Arquitectura (UNPHU, 1994); Doctorado en Hiroshima University (Japón, 2002); jurado de maestría en Columbia University (USA, 2010); profesor titular en la Universidad Iberoamericana (UNIBE) y coordinador de Innovación Arquitectónica (UNPHU). Ha publicado en el Journal of Architecture, Planning and Environmental Engineering (AIJ, Tokyo), Misawa Bauhaus Collection (Tokyo), Portal Docente UNIBE y revista AULA UNPHU. Miembro del Architectural Institute of Japan (AIJ, Tokyo), CODIA y SARD (Santo Domingo). Practica desde su firma FUTURA, Arquitectura Emergente SRL. 